\title{
The Antarctic Biodiversity Portal, an Online Ecosystem for Linking, Integrating and Disseminating Antarctic Biodiversity Information
}

\author{
Yi-Ming Gan ${ }^{\ddagger}$, Maxime Sweetlove ${ }^{\ddagger}$, Anton Van de Putte \\ ‡ Royal Belgian Institute of Natural Sciences, Brussels, Belgium
}

Corresponding author: Yi-Ming Gan (ymgan@naturalsciences.be), Maxime Sweetlove (msweetlove@naturalscience s.be), Anton Van de Putte (avandeputte@naturalsciences.be)

Received: 12 Jun 2019| Published: 18 Jun 2019

Citation: Gan Y, Sweetlove M, Van de Putte A (2019) The Antarctic Biodiversity Portal, an Online Ecosystem for Linking, Integrating and Disseminating Antarctic Biodiversity Information. Biodiversity Information Science and Standards 3: e37182. https://doi.org/10.3897/biss.3.37182

\section{Abstract}

The Antarctic Biodiversity portal (biodiversity.aq) is a gateway to a wide variety of Antarctic biodiversity information and tools. Launched in 2015 as the Scientific Committee on Antarctic Research (SCAR) - Marine Biodiversity Information Network (SCAR-MarBIN, scarmarbin.be) and the Register of Antarctic Marine Species (RAMS, marinespecies.org/ rams/), the system has grown in scope from purely marine to include terrestrial information.

Biodiversity.aq is a SCAR product, currently supported by Belspo (Belgian Science Policy) as one of the Belgian contributions to the European Lifewatch-European Research Infrastructure Consortium (Lifewatch-ERIC). The goal of Lifewatch is to provide access to: distributed observatories/sensor networks; interoperable databases, existing (data-) networks, using accepted standards; high performance computing (HPC) and grid power, including the use of the state-of-the-art of cloud and big data paradigm technologies; software and tools for visualization, analysis and modeling.

Here we provide an overview of the most recent advances in the biodiversity.aq online ecosystem, a number of use cases as well as an overview of future directions. Some of the most notable components are: 
- The Register of Antarctic Species (RAS, ras.biodiversity.aq) is a component of the Lifewatch Taxonomic Backbone and provides an authoritative and comprehensive list of names of marine and terrestrial species in Antarctica and the Southern Ocean. It serves as a reference guide for users to interpret taxonomic literature, as valid names and other names in use are both provided.

- Integrated Publishing Toolkit (IPT, ipt.biodiversity.aq) allows disseminating Antarctic biodiversity data into global initiatives such as the Ocean Biogeographic Information System (OBIS, obis.org) as Antarctic node of OBIS (Ant-OBIS, also formerly known as SCAR-MarBIN) and the Global Biodiversity Information Facility (GBIF, gbif.org) as Antarctic Biodiversity Information Facility (AntaBIF). Data that can be made available include metadata, species checklists, species occurrence data and more recently, sampling event-based data. Data from these international portals can be accessed through data.biodiversity.aq.

Through SCAR, Biodiversity.aq builds on an international network of expert that provide expert knowledge on taxonomy, species distribution,and ecology. It provides a strong and tested platform for sharing, integrating, discovering and analysing Antarctic biodiversity information originating from a variety of sources into a distributed system.

\section{Keywords}

Antarctic, biodiversity, data management

\section{Presenting author}

Yi-Ming Gan

\section{Presented at}

Biodiversity_Next 2019

\section{Funding program}

EU-Lifewatch

\section{Hosting institution}

Royal Belgian Institute for Natural Sciences 